\title{
FOKUS Dermatochirurgie: Der Blick über den Tellerrand lohnt sich
}

Gastherausgeber: Prof. Dr. Falk Bechara (Bochum)

Editorial

48 Dermatochirurgie: Der Blick über den Tellerrand lohnt sich FOKUS Bechara, F.G. (Bochum)

Übersichtsarbeit

50 Erfolgreiche Applikation von modifizierten Keystone-Lappen nach Hauttumorablation FOKUS

Huang, J. (Beijing); Chan Woo, K. (Soengnam); Wang, X.; Yumo, Z.; Nanze, Y.; Ru, Z.; Ming, B.; Xiao, L. (Beijing); Park, T. (Soengnam)

Wissenstransfer

56 Subunguales Melanom: Weniger invasives operatives Verfahren als Standard etablieren FOKUS

Schnabl-Scheu, S. (Tübingen)

58 Defektdeckung: Stufenweise Modifikation der Lappenpräparation bringt Vorteile FOKUS

Sand, M.; Quambusch, M. (Essen)

60 Varikose: Welche Komplikationen im Aufklärungsgespräch genannt werden sollten Stücker, M. (Bochum)

62 Psoriasis: Lokalisation beeinflusst die Krankheitslast Sondermann, W. (Essen)

65 Hautalterung: Über die Rolle von Fibroblasten-Wachstumsfaktoren Schulz, A. (Essen)

68 Chromoblastomykose: Mit der Kombination aus PDT und Antimykotika zum Erfolg

Lehmann, P. (Wuppertal)

DermaCampus

70 DermaCampus in den Zeiten von COVID-19

Mader, M. (Kamp-Lintfort)

71 Aus der Praxis: Operative Lasermedizin Hammes, S. (Greifswald)

72 Aus der Praxis: Arztberuf im Wandel - welche Herausforderungen erwarten uns? Operation Karriere 


\begin{tabular}{|c|c|}
\hline & Steckbrief Forschung \\
\hline 74 & $\begin{array}{l}7 \text { Fragen an Gewinnerin des 2. Publikationspreises im } \\
\text { Fachbereich Spezielle Dermatochirurgie } 2019 \text { der DGDC } \\
\text { Schnabl-Scheu, S. (Tübingen) }\end{array}$ \\
\hline \multirow[t]{2}{*}{76} & PharmaNews \\
\hline & Erfahrung aus der Praxis \\
\hline \multirow[t]{3}{*}{79} & $\begin{array}{l}\text { Akrales amelanotisches Melanom, das wie ein nicht heilendes arterielles } \\
\text { Ulcus erscheint }\end{array}$ \\
\hline & Cantwell, P.; Van Dam, H. (Perth) \\
\hline & Kaleidoskop \\
\hline 82 & $\begin{array}{l}\text { Spektrum Dermatologie - wissenswert, kompakt, anregend } \\
\text { Unabhängige Berichterstattung aus Wissenschaft und Praxis }\end{array}$ \\
\hline \multirow[t]{2}{*}{87} & Veranstaltungskalender \\
\hline & Seitenblicke \\
\hline 88 & $\begin{array}{l}\text { Wo gute Ideen herkommen } \\
\text { Walach, H. (Poznań/Berlin) }\end{array}$ \\
\hline 45 & Wissenschaftlicher Beirat \\
\hline 45 & Impressum \\
\hline
\end{tabular}

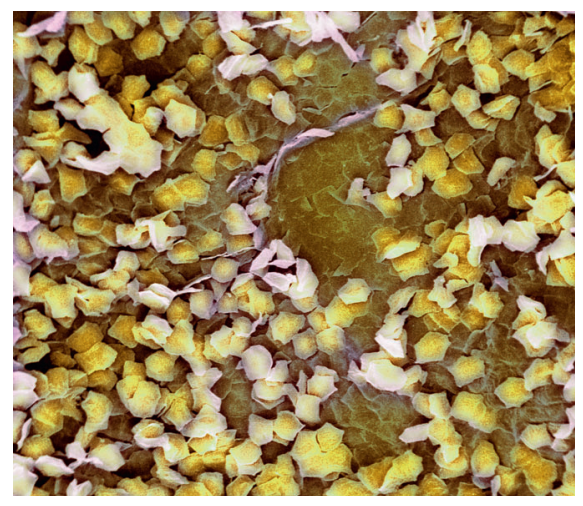

Titelbild

(o) Science Photo Library

Oberfläche der Haut. Rasterelektronenmikroskopische Aufnahme (REM) von Plattenepithelzellen auf der Haut.
45 Impressum

\section{Die nächste Ausgabe Kompass Dermatologie erscheint am 21. Juli 2020 mit dem Themenschwerpunkt «Dermato-Onkologie».}


Kompass

Dermatologie

\section{Herausgeber}

Prof. Dr. Andreas Körber (Essen)

\section{Fachbeirat}

Ästhetische Dermatologie

Dr. Welf Prager (Hamburg)

Dr. Andrea Schulz (Essen)

\section{Allergologie}

Prof. Dr. Uwe Gieler (Gießen)

Prof. Dr. Vera Mahler (Langen)

Prof. Dr. Claudia Traidl-Hoffmann (Augsburg)

\section{Berufs- und Umweltmedizin}

Prof. Dr. Vera Mahler (Langen)

\section{Dermato-Endokrinologie}

Dr. Eugenia Makrantonaki

(Wildeshausen/Ulm)

\section{Dermato-Onkologie \\ Dr. Jan Maschke (Köln) \\ PD Dr. Max Schlaak (München)}

\section{Entzündliche Hauterkrankungen}

Dr. Eugenia Makrantonaki (Wildeshausen/Ulm)

Dr. Sandra Philipp (Oranienburg)

Dr. Wiebke Sondermann (Essen)

\section{Geriatrische Dermatologie}

Prof. Dr. Markus Braun-Falco (München)

\section{Operative Dermatologie}

Dr. Welf Prager (Hamburg)

\section{Pädiatrische Dermatologie •}

Atopisches Ekzem

Prof. Dr. Regina Fölster-Holst (Kiel)

\section{Phlebologie}

Prof. Dr. Markus Stücker (Bochum)

\section{Photodermatologie}

Prof. Dr. Percy Lehmann (Wuppertal)

\section{Psychosomatische Dermatologie}

Prof. Dr. Uwe Gieler (Gießen)

\section{Versorgungsforschung}

Dr. Natalia Kirsten (Hamburg)
DermaCampus

Dr. Mario Mader (Kamp-Lintfort)

\section{IMPRESSUM}

ISSN gedruckte Edition: 2296-5424

ISSN Online-Edition: 2296-536X

Zeitschriften-Homepage: www.karger.com/kkd

Erscheinungsweise: Karger Kompass Dermatologie, Jahrgang 8, 2020, erscheint mit vier Ausgaben.

Copyright: $\odot 2020$ by S. Karger Verlag für Medizin und Naturwissenschaften GmbH, Freiburg i.Br. (Deutschland). Die Zeitschrift sowie alle in ihr enthaltenen einzelnen Beiträge und Abbildungen sind urheberrechtlich geschützt. Jede Verwertung, die nicht ausdrücklich vom Urheberrechtsgesetz zugelassen ist, bedarf der vorherigen Zustimmung des Verlags. Das gilt insbesondere für Vervielfältigungen, Bearbeitungen, Übersetzungen, Mikroverfilmungen und die Einspeicherung und Verarbeitung in elektronischen Systemen.
Disclaimer: Die in dieser Publikation enthaltenen Aussagen und Daten sind ausschließlich die der einzelnen Autoren und Mitwirkenden, und nicht die des Verlags und der Herausgeber. Das Erscheinen von Anzeigen stellt keine Gewähr, Bestätigung oder Zustimmung für die beworbenen Produkte oder Leistungen bzw. für deren Wirksamkeit, Qualität oder Sicherheit dar. Der Verlag und die Herausgeber lehnen jegliche Verantwortung für Schäden an Personen oder Eigentum ab, die mit den Ideen, Methoden, Anleitungen oder Produkten, die im Inhalt oder den Anzeigen beschrieben werden, in Verbindung stehen.

\section{Abonnementpreise:}

(Jahresbezugspreis für ein volles Kalenderjahr)

Print: $\quad$ EUR $90 \mathrm{zzgl}$. Versandkosten

Online: EUR 90

Kombiniert (print+online):

$$
\text { EUR } 135 \text { zzgl. Versandkosten }
$$

Versandkosten: EUR 11 (für Europa), USD 14 (Übersee, USA, Lateinamerika), CHF 14 (Rest of World)

Der Bezugspreis für Einzelhefte beträgt EUR 20 zzgl. Versandkosten.

\section{Verlag:}

S. Karger Verlag für Medizin und

Naturwissenschaften $\mathrm{GmbH}$

Schnewlinstraße 12, 79098 Freiburg (Deutschland)

Tel. +49761 4520 70, Fax +497614520714

www.karger.de, information@karger.com

Corporate Sales Manager:

Ellen Zimmermann

Tel. +497614520717

e.zimmermann@karger.com

Redaktion:

Christoph Habel

Tel. +497614520728

c.habel@karger.com

V.i.S.d.P.: Fritz Koller

Satz: S. Karger AG, Basel

Druck:

PRINTEC OFFSET medienhaus, 34123 Kassel, Germany Gedruckt auf säurefreiem und alterungsbeständigem Papier (ISO 9706)

Beilagenhinweis:

Diese Ausgabe enthält eine Beilage der Almirall Hermal GmbH. Verbreitung nur in Deutschland 